2017 Управление, вычислительная техника и информатика № 41

УДК 336.51

DOI: $10.17223 / 19988605 / 41 / 5$

\title{
G.A. Medvedev \\ ON YIELD CURVES OF THE EUROPEAN CENTRAL BANK
}

In this paper it is showed that the yield curve of the European Central Bank (ECB) does not satisfy the no arbitrage conditions. For this it is need to add one more term to yield curve. As the state variables, it is necessary to choose a fourdimensional diffusion Gaussian process. Fifth factor is determined from the no arbitrage conditions. The version of the modification of the yield curve proposed in the paper differs from the earlier proposed modifications in order to ensure the absence of arbitrage opportunities.

Keywords: yield curve; forward curve; Nelson-Siegel-Svensson model; no arbitrage conditions.

In June 1996, the Bank for International Settlements (BIS, Basel) agreed that the central banks of Europe should submit their data to the BIS for the calculation of zero-coupon yield curves and model parameter estimates. It was found that most European banks use the Nelson - Siegel approach (Italy and Finland) or its modification of Svensson (Belgium, Germany, Spain, Norway, France, Switzerland and Sweden) to model yield curves [1].

Unfortunately, the Nelson - Siegel - Svensson (NSS) approach does not give recommendations on how to determine the parameters of the model (only to evaluate them) and does not explain in any way whether such a model is arbitrage-free. Therefore, it makes sense to consider this problem.

\section{Dynamics of parameters change in yield (spot) rate and forward curve}

Statistics of the Directorate General of the European Central Bank (ECB) publishes the euro zone's yield curves every TARGET working day at 12 noon on Central European time [2]. In the ECB technical note [3] formulas are given for calculating three types of yield curves: the yield curve, the forward curve and the nominal yield curve. Formulas for the first two of them have the form:

- yield (spot) rate

$$
y(\tau)=\beta_{0}+\beta_{1}\left(\frac{1-e^{-\tau / \tau_{1}}}{\tau / \tau_{1}}\right)+\beta_{2}\left(\frac{1-e^{-\tau / \tau_{1}}}{\tau / \tau_{1}}-e^{-\tau / \tau_{1}}\right)+\beta_{3}\left(\frac{1-e^{-\tau / \tau_{2}}}{\tau / \tau_{2}}-e^{-\tau / \tau_{2}}\right),
$$

- forward curve

$$
f(\tau)=\beta_{0}+\beta_{1} e^{-\tau / \tau_{1}}+\beta_{2} \frac{\tau}{\tau_{1}} e^{-\tau / \tau_{1}}+\beta_{3} \frac{\tau}{\tau_{2}} e^{-\tau / \tau_{2}} .
$$

Here $\tau$ is a term to maturity, $\beta_{i}, \tau_{i}$ are the parameters that are estimated on the market data. In Fig. 1 for example, the yield curve and the forward curve from the ECB website on June 1, 2017 presented.

On the ECB website the curves are shown for term to maturities $\tau$ from 0 to 30 years. In contrast to this, in order to show these curves "whole" for the entire range of values of $\tau$ from 0 to $\infty$, on Fig. 1 we used the mapping of the positive semiaxis $\tau \in(0, \infty)$ of terms to maturity to an unit interval of new variable $u \in(0,1)$ by means of nonlinear transformation $u=1-e^{-\rho \tau}$. The parameter $\rho$ was chosen in such a way that the interval of maturities $\tau \in(0,30)$ corresponds to the values of $u \in(0,0.9)$, i. e. $\rho=\ln 10 / 30=0.07675$. It is relevant to note here that the curves always start from one common point $y(0 \mid \beta)=f(0 \mid \beta)=r(t)$, current value of shortterm interest rate, and for $\tau \rightarrow \infty$ always tend to one common limit value $y(\infty \mid \beta)=f(\infty \mid \beta)=\beta_{0}$.

Because the yield curve is to be time-varying (as it obviously is) and following [4], [5] let us here recognize that the Nelson-Siegel-Svensson (NSS) parameters $\beta_{0}, \beta_{1}, \beta_{2}, \beta_{3}$ must be time-varying too. 


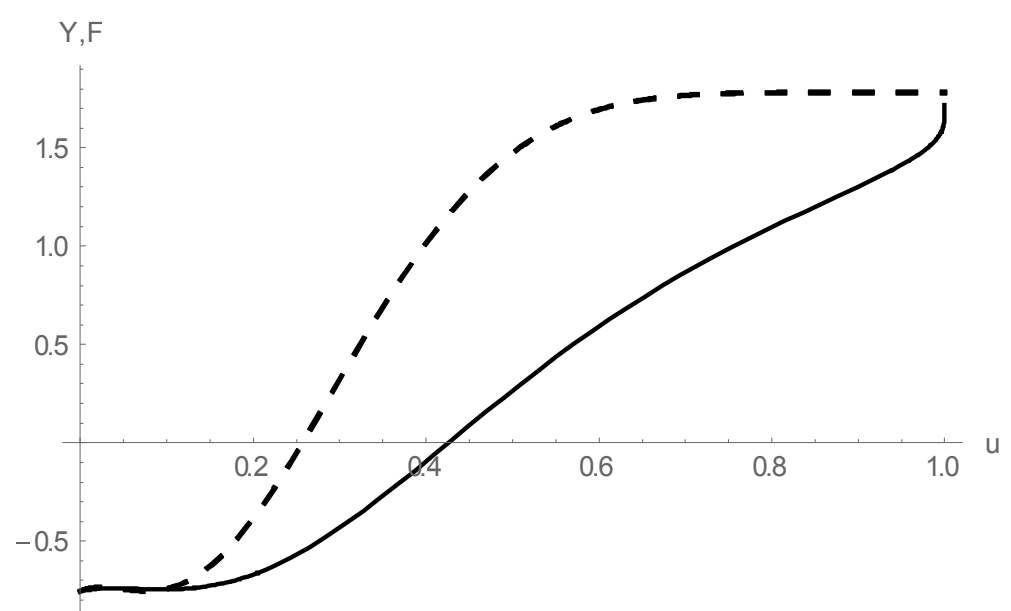

Fig. 1. The yield curve (the lower curve) and the forward curve (the upper curve) from the ECB website on June 1, 2017. Parameter values: $\beta_{0}=1.7810, \beta_{1}=-2.5350$, $\beta_{2}=23.2948, \beta_{3}=-27.6452, \tau_{1}=1.5822, \tau_{2}=1.7081$

Thus in the NSS yield models the yield curve $y(\tau) \equiv y\left(\tau \mid \beta_{0}, \beta_{1}, \beta_{2}, \beta_{3}\right)$ and the forward curve $f(\tau) \equiv f\left(\tau \mid \beta_{0}, \beta_{1}\right.$, $\beta_{2}, \beta_{3}$ ) are determined by following relations

$$
\begin{aligned}
& y(\tau \mid \beta)=\beta_{0}(\tau)+\beta_{1}(t) \frac{1-e^{-\gamma \tau}}{\gamma \tau}+\beta_{2}(t)\left(\frac{1-e^{-\gamma \tau}}{\gamma \tau}-e^{-\gamma \tau}\right)+\beta_{3}(t)\left(\frac{1-e^{-\delta \tau}}{\delta \tau}-e^{-\delta \tau}\right), \\
& f(\tau \mid \beta)=\left(\tau \beta_{0}(\tau)\right)^{\prime}+\beta_{1}(t) \exp (-\gamma \tau)+\beta_{2}(t) \gamma \tau \exp (-\gamma \tau)+\beta_{3}(t) \delta \tau \exp (-\delta \tau) .
\end{aligned}
$$

For convenience, some rename have been made here: $\gamma \equiv 1 / \tau_{1}, \delta \equiv 1 / \tau_{2}$. The parameters $\beta_{0}, \beta_{1}, \beta_{2}, \beta_{3}$ now depend on the current time $t$. The dependence of the parameter $\beta_{0}$ on the current time is somewhat different: $\beta_{0}(T-t) \equiv \beta_{0}(\tau)$, where $T$ is the maturity date. The need for this will be clarified later. Prime means derivative with respect to the term to maturity $\tau=T-t$. In this case the state of the market can be described by a set of parameters $\left\{\beta_{1}(t), \beta_{2}(t), \beta_{3}(t)\right\}$ and each of the parameters considered as a component of the state vector $\beta(t)$. Whether such yield curves satisfy the no arbitrage conditions? In order to clarify this it at first it is need to determine how the dynamics of the state variables $\beta(t)$ are described. At present the diffusion stochastic processes are most often used to describe the dynamics of these variables, the mathematical model of which is the stochastic differential equation

$$
d \beta(t)=\mu(\beta) d t+\sigma(\beta) d W(t)
$$

where $\mu(\beta)$ is a state drift vector, $\sigma(\beta)$ is a state vector volatility matrix, $W(t)$ is a vector of Wiener processes. In order to the arbitrage opportunities to be absent, it is necessary that the price of the zero-coupon bond $P(t, T$, $\beta)=\exp [-\tau y(\tau, \beta)]$ on a fixed date $t$ satisfies the so-called term structure equation [6]:

$$
\begin{gathered}
\frac{\partial P(t, T, \beta)}{\partial t}+\mu(\beta)^{\mathrm{T}} \frac{\partial P(t, T, \beta)}{\partial \beta}+\frac{1}{2} \operatorname{tr}\left(\sigma(\beta)^{\mathrm{T}} \frac{\partial^{2} P(t, T, \beta)}{\partial \beta^{2}} \sigma(\beta)\right)-r(t) P(t, T, \beta)= \\
=\lambda(t, \beta)^{\mathrm{T}} \sigma(\beta)^{\mathrm{T}} \frac{\partial P(t, T, \beta)}{\partial \beta} .
\end{gathered}
$$

Here $r(t)$ is short-term interest rate, $\lambda(t, \beta)$ is a market risk price vector on some fixed date $t$. The equation must be supplemented by boundary conditions: $\beta_{0}(0)=0, y(0, \beta)=r(t)$ (because of definition of short-term interest rate). It is possibly establish an more explicit relationship between the state variables $\left\{\beta_{1}(t), \beta_{2}(t), \beta_{3}(t)\right\}$ and short-term interest rate from limiting relation $\lim _{T \rightarrow t} y(T-t, \beta)=r(t)$. Indeed for any time $t$

$$
\begin{aligned}
\lim _{T \rightarrow t} y(T-t, \beta)= & \lim _{\tau \rightarrow 0}\left[\beta_{0}(\tau)+\beta_{1}(t) \frac{1-e^{-\gamma \tau}}{\gamma \tau}+\beta_{2}(t)\left(\frac{1-e^{-\gamma \tau}}{\gamma \tau}-e^{-\gamma \tau}\right)+\right. \\
& \left.+\beta_{3}(t)\left(\frac{1-e^{-\delta \tau}}{\delta \tau}-e^{-\delta \tau}\right)\right]=\beta_{1}(t)=r(t),
\end{aligned}
$$


as $\beta_{0}(0)=0$. Thus the economical meaning of component $\beta_{1}(t)$ of the state vector $\beta(t)$ is short-term interest rate $r(t)$.

Let us introduce additional notation facilitating analysis corresponding to the state vector $\beta(t)=\left(\beta_{1}(t)\right.$, $\left.\beta_{2}(t), \beta_{3}(t)\right)^{\mathrm{T}}$. Since the yield curves characterize the term structure of interest rates for some fixed value of the current time $t$, we will consider $t$ to be a fixed value that does not change in the analysis, and for brevity we omit it in the notation. We introduce the vector of the term structure functions $B(\tau)=\left(B_{1}(\tau), B_{2}(\tau), B_{3}(\tau)\right)^{\mathrm{T}}$ :

$$
B_{1}(\tau)=\frac{1-e^{-\gamma \tau}}{\gamma}, B_{2}(\tau)=\left(\frac{1-e^{-\gamma \tau}}{\gamma}-\tau e^{-\gamma \tau}\right), B_{3}(\tau)=\left(\frac{1-e^{-\delta \tau}}{\delta}-\delta e^{-\delta \tau}\right) .
$$

Then the yield curve $y(\tau, \beta)$ and forward curve $f(\tau, \beta)$ in (3), (4) will have representations

$$
y(\tau, \beta)=\left[\tau \beta_{0}(\tau)+\beta(t)^{\mathrm{T}} B(\tau)\right] / \tau, f(\tau, \beta)=\left[\tau \beta_{0}(\tau)+\beta(t)^{\mathrm{T}} B(\tau)\right]^{\prime} .
$$

Because the price of the zero-coupon bond are represented in the form $P(t, T, \beta)=\exp [-\tau y(\tau, \beta)]$ it is more convenient to write the term structure equation not for the price $P(t, T, \beta)$ but for yield $y(\tau, \beta)$

$$
\frac{\partial(\tau y(\tau, \beta))}{\partial \tau}+\tau[\sigma(\beta) \lambda(\beta)-\mu(\beta)]^{\mathrm{T}} \frac{\partial y(\tau, \beta)}{\partial \beta}+\frac{\tau}{2} \operatorname{tr}\left(\sigma(\beta)^{\mathrm{T}}\left[\tau \frac{\partial y(\tau, \beta)}{\partial \beta}\left(\frac{\partial y(\tau, \beta)}{\partial \beta}\right)^{\mathrm{T}}-\frac{\partial^{2} y(\tau, \beta)}{\partial \beta^{2}}\right] \sigma(\beta)\right)-r=0 .
$$

Taking into account that $y(\tau, \beta)=\left[\tau \beta_{0}(\tau)+\beta^{\mathrm{T}} B(\tau)\right] / \tau$, we can write

$$
\frac{\partial\left(\tau \beta_{0}(\tau)\right)}{\partial \tau}+\beta^{\mathrm{T}} \frac{\partial B(\tau)}{\partial \tau}+[\sigma(\beta) \lambda(\beta)-\mu(\beta)]^{\mathrm{T}} B(\tau)+\frac{1}{2} B(\tau)^{\mathrm{T}} \sigma(\beta) \sigma(\beta)^{\mathrm{T}} B(\tau)=r
$$

Consider the class of volatility matrices $\sigma(\beta)$ that do not depend on the current state of the market, i.e. $\sigma(\beta)=\sigma$. In this case the market risk price vector $\lambda(\beta)$ does not depend on the current state of the market too, $\lambda(\beta)=\lambda$. Such models of the dynamics of state variables $\beta$ can naturally be called extended Vasiček models. Then the term structure equation reduces to following

$$
\frac{\partial\left(\tau \beta_{0}(\tau)\right)}{\partial \tau}+\lambda^{\mathrm{T}} \sigma^{\mathrm{T}} B(\tau)+\frac{1}{2} B(\tau)^{\mathrm{T}} \sigma \sigma^{\mathrm{T}} B(\tau)=-\beta^{\mathrm{T}} \frac{\partial B(\tau)}{\partial \tau}+\mu(\beta)^{\mathrm{T}} B(\tau)+r .
$$

The left-hand side of the equation does not depend on the state variables $\beta$, while on the right-hand side each term depends on them (we recall that $r=\beta_{1}$ ). Since this equality must hold for any vectors $\beta$ and for any terms to maturity $\tau$, it splits into two equations: the left-hand side gives the equation for the function $\beta_{0}(\tau)$, and the right-hand side determines the drift function $\mu(\beta)$.

$$
\begin{gathered}
\frac{\partial\left(\tau \beta_{0}(\tau)\right)}{\partial \tau}+\lambda^{\mathrm{T}} \sigma^{\mathrm{T}} B(\tau)+\frac{1}{2} B(\tau)^{\mathrm{T}} \sigma \sigma^{\mathrm{T}} B(\tau)=c(\tau), \\
\mu(\beta)^{\mathrm{T}} B(\tau)=c(\tau)+\beta^{\mathrm{T}} \frac{\partial B(\tau)}{\partial \tau}-\beta_{1},
\end{gathered}
$$

where so far $c(\tau)$ is an undefined function that can be zero. Thus

$$
\beta_{0}(\tau)=\frac{1}{\tau} \int_{0}^{\tau}\left(c(s)-\lambda^{\mathrm{T}} \sigma^{\mathrm{T}} B(s)-\frac{1}{2} B(s)^{\mathrm{T}} \sigma \sigma^{\mathrm{T}} B(s)\right) d s .
$$

As for the drift function $\mu(\beta)$, since the right-hand side (12) is an affine function, the drift function must also be an affine function too. Define it as $\mu(\beta)=K \beta+\xi$ where the matrix $K$ and the vector $\xi$ are do not depend on $\tau$ and satisfy to relation (12) or

$$
\xi^{\mathrm{T}} B(\tau)-c(\tau)=\beta^{\mathrm{T}}\left(\frac{\partial B(\tau)}{\partial \tau}-K^{\mathrm{T}} B(\tau)\right)-\beta_{1} .
$$

The left-hand side of equality (14) defines a function $\mathrm{c}(\tau)$ : $\mathrm{c}(\tau)=\xi^{\mathrm{T}} B(\tau)$. The right-hand side of equality (14) defines the requirement which the matrix $K$ must satisfy to be fulfilled the no arbitrage condition:

$$
\beta^{\mathrm{T}}\left(\frac{\partial B(\tau)}{\partial \tau}-K^{\mathrm{T}} B(\tau)\right)=\beta_{1}
$$


In the scalar form condition (15) is equivalent to the system of equations

$$
\begin{gathered}
e^{-\gamma \tau}-k_{11} \frac{1-e^{-\gamma \tau}}{\gamma}-k_{21}\left(\frac{1-e^{-\gamma \tau}}{\gamma}-\tau e^{-\gamma \tau}\right)-k_{31}\left(\frac{1-e^{-\delta \tau}}{\delta}-\tau e^{-\delta \tau}\right)=1, \\
\gamma \tau e^{-\gamma \tau}-k_{12} \frac{1-e^{-\gamma \tau}}{\gamma}-k_{22}\left(\frac{1-e^{-\gamma \tau}}{\gamma}-\tau e^{-\gamma \tau}\right)-k_{32}\left(\frac{1-e^{-\delta \tau}}{\delta}-\tau e^{-\delta \tau}\right)=0, \\
\delta \tau e^{-\delta \tau}-k_{13} \frac{1-e^{-\gamma \tau}}{\gamma}-k_{23}\left(\frac{1-e^{-\gamma \tau}}{\gamma}-\tau e^{-\gamma \tau}\right)-k_{33}\left(\frac{1-e^{-\delta \tau}}{\delta}-\tau e^{-\delta \tau}\right)=0,
\end{gathered}
$$

where $\left\{k_{i j}\right\}$ are the elements of matrix $K$. The first equation is satisfied by $k_{11}=-\gamma, k_{21}=k_{31}=0$. The second equation is satisfied by $k_{12}=\gamma, k_{22}=-\gamma, k_{32}=0$. However in order to satisfy the third equality there does not exist any constants $\left\{k_{13}, k_{23}, k_{33}\right\}$. Therefore if the volatility matrix $\sigma$ does not depend on the state variable $\beta$, the yield curve $y(\tau, \beta)$ does not satisfy the no arbitrage condition (15). Thus, the yield term structure of the ECB (1) does not ensure the fulfillment of no arbitrage conditions if the volatility matrices $\sigma(\beta)$ do not depend on the current state of the market, i.e. $\sigma(\beta)=\sigma$.

Suppose now that the volatility matrix and the corresponding vector of market risk prices are such that matrix $\sigma(\beta) \sigma^{\mathrm{T}}(\beta)$ and vector $\sigma(\beta) \lambda(\beta)$ are affine functions of the state variable $\beta$, i.e.

$$
\sigma(\beta) \sigma^{\mathrm{T}}(\beta)=\omega_{0}+\sum_{i} \omega_{i} \beta_{i}, \sigma(\beta) \lambda(\beta)=\lambda_{0}+\lambda_{1} \beta,
$$

where $\omega_{0}, \omega_{i}, \lambda_{1}$ are matrices and $\lambda_{0}$ is vector with constant elements. Note that matrices $\left\{\omega_{i}\right\}$ are symmetric. Such models of the dynamics of state variables $\beta$ are the Duffie-Kan models (with square-root volatility) [7]. Then equation of term structure (9) becomes

$$
\frac{\partial\left(\tau \beta_{0}(\tau)\right)}{\partial \tau}+\beta^{\mathrm{T}} \frac{\partial B(\tau)}{\partial \tau}+\left[\lambda_{0}+\lambda_{1} \beta-\mu(\beta)\right]^{\mathrm{T}} B(\tau)+\frac{1}{2} B(\tau)^{\mathrm{T}} \omega_{0} B(\tau)+\frac{1}{2} \sum_{i} B(\tau)^{\mathrm{T}} \omega_{i} B(\tau) \beta_{i}=r .
$$

From the form of equation (17) it follows that the drift function $\mu(\beta)$ must be an affine too, $\mu(\beta)=K \beta+\xi$. Then equation (17) can be written as follows

$$
\frac{\partial\left(\tau \beta_{0}(\tau)\right)}{\partial \tau}+\left(\lambda_{0}-\xi\right)^{\mathrm{T}} B(\tau)+\frac{1}{2} B(\tau)^{\mathrm{T}} \omega_{0} B(\tau)=\beta^{\mathrm{T}}\left(\left(K-\lambda_{1}\right)^{\mathrm{T}} B(\tau)-\frac{\partial B(\tau)}{\partial \tau}-\varphi(\tau)\right)+\beta_{1} .
$$

Here for brevity it is denoted $\varphi(\tau)$ is vector with components $\varphi_{i}(\tau)=0.5 B(\tau)^{\mathrm{T}} \omega_{i} B(\tau)$. In equation (18) the lefthand side does not depend on the state variables $\beta$, while each term on the right-hand side of the equation depends on these variables. This makes it possible, on the one hand, to determine the function $\beta_{0}(\tau)$ and, on the other hand, to find the no arbitrage conditions to which must satisfy the set of elements of the matrices $\mathrm{K}, \omega_{1}$, $\omega_{2}, \omega_{3}, \lambda_{1}$. For this from equation (18) it is possible to obtain two following equalities

$$
\begin{gathered}
\beta_{0}(\tau)=\frac{1}{\tau} \int_{0}^{\tau}\left(\left(\lambda_{0}-\xi\right)^{\mathrm{T}} B(s)-\frac{1}{2} B(s)^{\mathrm{T}} \omega_{0} B(s)\right) d s, \\
\beta^{\mathrm{T}}\left(\frac{\partial B(\tau)}{\partial \tau}-\left(K-\lambda_{1}\right)^{\mathrm{T}} B(\tau)+\varphi(\tau)\right)=\beta_{1} .
\end{gathered}
$$

If there exist matrices $\mathrm{K}, \omega_{1}, \omega_{2}, \omega_{3}, \lambda_{1}$ that satisfy the equality (20) then for the yield curve (1) the no-arbitrage conditions satisfy too. The equality (20) must hold for any set of state variables $\left\{\beta_{1}, \beta_{2}, \beta_{3}\right\}$ and any term to maturity $\tau \in(0, \infty)$. Therefore, equating to zero the coefficients for different variables $\beta_{i}$, it is possible to obtain three following equalities

$$
\begin{gathered}
\frac{\partial B_{1}(\tau)}{\partial \tau}-B(\tau)^{\mathrm{T}}\left(K-\lambda_{1}\right)_{1}+0.5 B(\tau)^{\mathrm{T}} \omega_{1} B(\tau)=1, \\
\frac{\partial B_{2}(\tau)}{\partial \tau}-B^{\mathrm{T}}(\tau)\left(K-\lambda_{1}\right)_{2}+0.5 B(\tau)^{\mathrm{T}} \omega_{2} B(\tau)=0, \\
\frac{\partial B_{3}(\tau)}{\partial \tau}-B^{\mathrm{T}}(\tau)\left(K-\lambda_{1}\right)_{3}+0.5 B(\tau)^{\mathrm{T}} \omega_{3} B(\tau)=0,
\end{gathered}
$$


where $\left(K-\lambda_{1}\right)_{i}$ is $i$-th column of matrix $\left(K-\lambda_{1}\right)$. If at least one of these three equalities is not satisfied, then the yield curve ECB (1) does not ensure the performance of the no arbitrage condition. Let us check the third equality. We substitute into this equality the explicit form of the vectors and matrices entering into it. As a result, the left-hand side of the equality will be a linear combination of thirteen linearly independent functions

$$
e^{-\tau \gamma}, e^{-2 \tau \gamma}, e^{-\tau \delta}, e^{-2 \tau \delta}, \tau e^{-\tau \gamma}, \tau e^{-2 \tau \gamma}, \tau e^{-\tau \delta}, \tau e^{-2 \tau \delta}, \tau^{2} e^{-2 \tau \gamma}, \tau^{2} e^{-2 \tau \delta}, e^{-\tau \gamma-\tau \delta}, \tau e^{-\tau \gamma-\tau \delta}, \tau^{2} e^{-\tau \gamma-\tau \delta},
$$

and constant. In order for this linear combination to be zero for any term to maturity $\tau$, the coefficients at all linearly independent functions and the constant must be equated to zero. This leads to unrealizable equality (if $\delta>0): \tau \delta \exp [-\tau \delta]=0$. It means that the ECB term structure of interest rate (1) does not ensure the fulfillment of the no arbitrage condition in this case too.

\section{Modification the yield curve}

The question arises how to modify the yield curve (1) so that it satisfies the no-arbitrage condition. We add one more term to the expression (3):

$$
y(\tau, \beta)=\beta_{0}(\tau)+\beta_{1}(t) \frac{1-e^{-\gamma \tau}}{\gamma \tau}+\beta_{2}(t)\left(\frac{1-e^{-\gamma \tau}}{\gamma \tau}-e^{-\gamma \tau}\right)+\beta_{3}(t)\left(\frac{1-e^{-\delta \tau}}{\delta \tau}-e^{-\delta \tau}\right)+\beta_{4}(t) \frac{1-e^{-\delta \tau}}{\delta \tau},
$$

which corresponds to the fact that an additional state variable $\beta_{4}$ is introduced together with the additional function of the term structure corresponding to it $B_{4}(\tau)=\left(1-e^{-\delta \tau}\right) / \delta$. Then the state vector $\beta$ will have four components $\left(\beta_{1}, \beta_{2}, \beta_{3}, \beta_{4}\right)^{\mathrm{T}}$ and the vector of the term structure functions $B(\tau)=\left(B_{1}(\tau), B_{2}(\tau), B_{3}(\tau), B_{4}(\tau)\right)^{\mathrm{T}}$ will have following components

$$
B_{1}(\tau)=\frac{1-e^{-\gamma \tau}}{\gamma}, B_{2}(\tau)=\left(\frac{1-e^{-\gamma \tau}}{\gamma}-\tau e^{-\gamma \tau}\right), B_{3}(\tau)=\left(\frac{1-e^{-\delta \tau}}{\delta}-\tau e^{-\delta \tau}\right), B_{4}(\tau)=\frac{1-e^{-\delta \tau}}{\delta} .
$$

The yield curve $y(\tau, \beta)$ and forward curve $f(\tau, \beta)$ in this case will have representations (8) too

$$
y(\tau, \beta)=\left[\tau \beta_{0}(\tau)+\beta(t)^{\mathrm{T}} B(\tau)\right] / \tau, f(\tau, \beta)=\left[\tau \beta_{0}(\tau)+\beta(t)^{\mathrm{T}} B(\tau)\right]^{\prime} .
$$

The term structure equation for yield $y(\tau, \beta)$ will be the same as (9). However, the short-term interest rate $r$ here will be determined differently. Indeed for any time $t$ we have $\lim _{T \rightarrow t} y(T-t, \beta)=r(t)$.

$$
\begin{gathered}
\lim _{T \rightarrow t} y(T-t, \beta)=\lim _{\tau \rightarrow 0}\left[\beta_{0}(\tau)+\beta_{1}(t) \frac{1-e^{-\gamma \tau}}{\gamma \tau}+\beta_{2}(t)\left(\frac{1-e^{-\gamma \tau}}{\gamma \tau}-e^{-\gamma \tau}\right)+\right. \\
\left.+\beta_{3}(t)\left(\frac{1-e^{-\delta \tau}}{\delta \tau}-e^{-\delta \tau}\right)+\beta_{4}(t) \frac{1-e^{-\delta \tau}}{\delta \tau}\right]=\beta_{1}(t)+\beta_{4}(t)=r(t),
\end{gathered}
$$

as $\beta_{0}(0)=0$. Thus the economical meaning of sum component $\beta_{1}(t)$ and $\beta_{4}(t)$ of the state vector $\beta(t)$ is shortterm interest rate $r(t)$. Further, for volatility matrices $\sigma(\beta)$ that do not depend on the current state $\beta$ of the market, i.e. $\sigma(\beta)=\sigma$, the equations (10) $-(11)$ will be true in this case too. And for $\mu(\beta)=K \beta+\xi$ equation (15) can be written as follows

$$
\beta^{\mathrm{T}}\left(\frac{\partial B(\tau)}{\partial \tau}-K^{\mathrm{T}} B(\tau)\right)=\beta_{1}+\beta_{4}
$$

So system (16) becomes

$$
\begin{gathered}
e^{-\gamma \tau}-k_{11} \frac{1-e^{-\gamma \tau}}{\gamma}-k_{21}\left(\frac{1-e^{-\gamma \tau}}{\gamma}-\tau e^{-\gamma \tau}\right)-k_{31}\left(\frac{1-e^{-\delta \tau}}{\delta}-\tau e^{-\delta \tau}\right)-k_{41} \frac{1-e^{-\delta \tau}}{\delta}=1, \\
\gamma \tau e^{-\gamma \tau}-k_{12} \frac{1-e^{-\gamma \tau}}{\gamma}-k_{22}\left(\frac{1-e^{-\gamma \tau}}{\gamma}-\tau e^{-\gamma \tau}\right)-k_{32}\left(\frac{1-e^{-\delta \tau}}{\delta}-\tau e^{-\delta \tau}\right)-k_{42} \frac{1-e^{-\delta \tau}}{\delta}=0, \\
\delta \tau e^{-\delta \tau}-k_{13} \frac{1-e^{-\gamma \tau}}{\gamma}-k_{23}\left(\frac{1-e^{-\gamma \tau}}{\gamma}-\tau e^{-\gamma \tau}\right)-k_{33}\left(\frac{1-e^{-\delta \tau}}{\delta}-\tau e^{-\delta \tau}\right)-k_{43} \frac{1-e^{-\delta \tau}}{\delta}=0,
\end{gathered}
$$




$$
e^{-\delta \tau}-k_{14} \frac{1-e^{-\gamma \tau}}{\gamma}-k_{24}\left(\frac{1-e^{-\gamma \tau}}{\gamma}-\tau e^{-\gamma \tau}\right)-k_{34}\left(\frac{1-e^{-\delta \tau}}{\delta}-\tau e^{-\delta \tau}\right)-k_{44} \frac{1-e^{-\delta \tau}}{\delta}=1 .
$$

This system is satisfied for following matrix $K$

$$
K=\left(\begin{array}{cccc}
-\gamma & \gamma & 0 & 0 \\
0 & -\gamma & 0 & 0 \\
0 & 0 & -\delta & 0 \\
0 & 0 & \delta & -\delta
\end{array}\right)
$$

The variable $\beta_{0}(\tau)$ is determined by formula (19) where $\omega_{0} \equiv \sigma \sigma^{\mathrm{T}}$ :

$$
\beta_{0}(\tau)=\frac{1}{\tau} \int_{0}^{\tau}\left(\left(\lambda_{0}-\xi\right)^{\mathrm{T}} B(s)-\frac{1}{2} B(s)^{\mathrm{T}} \sigma \sigma^{\mathrm{T}} B(s)\right) d s .
$$

This variable determines the long-term yield interest rate for big values $\tau$. In particular

$$
\begin{aligned}
\lim _{\tau \rightarrow \infty} y(\tau, \beta)=\lim _{\tau \rightarrow \infty} & \beta_{0}(\tau)=\frac{\lambda_{01}+\lambda_{02}-\xi_{1}-\xi_{2}}{\gamma}+\frac{\lambda_{03}+\lambda_{04}-\xi_{3}-\xi_{4}}{\delta}- \\
& -\frac{1}{2} \sum_{i=1}^{4}\left(\frac{\sigma_{i 1}+\sigma_{i 2}}{\gamma}+\frac{\sigma_{i 3}+\sigma_{i 4}}{\delta}\right)^{2} .
\end{aligned}
$$

Here $\xi_{\mathrm{i}}, \lambda_{0 \mathrm{i}}, \sigma_{\mathrm{ij}}$ are the elements of $\xi, \lambda_{0}, \sigma$ respectively.

Thus, the yield curve (22) ensures fulfillment of the no arbitrage condition unlike the ECB yield curve (1). The verification of the no arbitrage condition by the Duffie - Kan model (with square-root volatility) leads to the fact that matrices $\omega_{1}=\omega_{2}=\omega_{3}=\omega_{4}=\lambda_{1}=0$ (the derivation of this here is not presented because of the bulkiness) and the model reduces to the four-factor Vasiček model, which we just considered.

The four-factor Vasiček model free of arbitrage generates a yield curve that completely coincides with the yield curve (22). So the NSS model (plus additional term as in (23)) is a particular case of the affine model, when the dynamics of the state variables $\beta=\left(\beta_{1}, \beta_{2}, \beta_{3}, \beta_{4}\right)^{\mathrm{T}}$ are determined by the equation

$$
d \beta(t)=K(\beta(t)-\theta) d t+\sigma d W(t),
$$

where matrix $K$ is the same as (23), matrix $\sigma$ has constant elements, and $\theta=-K^{-1} \xi$ is a vector of the stationary mathematical expectations of state variables $\beta$. Within the class of affine systems of the term structure of interest rates the price of a zero-coupon bond is presented in the form $P(\tau, \beta)=\exp \left\{A(\tau)-\beta^{\mathrm{T}} B(\tau)\right\}$, where function $A(\tau)$ and vector $B(\tau)$ satisfy the initial conditions: $A(0)=0$ и $B(0)=0$.

Note that for a bond price in this form, the short-term interest rate (1) takes the form

$$
y(0, \beta)=\lim _{\tau \rightarrow 0} \frac{-\ln P(\tau, \beta)}{\tau}=\lim _{\tau \rightarrow 0} \frac{\beta^{\mathrm{T}} B(\tau)-A(\tau)}{\tau}=\left.\beta^{\mathrm{T}} \frac{d B(\tau)}{d \tau}\right|_{\tau=0}-\left.\frac{d A(\tau)}{d \tau}\right|_{\tau=0}=\beta^{\mathrm{T}} B^{\prime}(0)-A^{\prime}(0),
$$

and is also an affine function of the vector $\beta$. Note that the state of the financial market is usually characterized by the values of interest rates, in other words, the components of the vector $\beta$ are quantities that have the meaning of interest rates. When interest rates are zero, there is no bond yield, so in (31) we should put $A^{\prime}(0)=0$. We denote $B^{\prime}(0)=\phi$. The vector $\phi=\left(\phi_{1}, \phi_{2}, \phi_{3}, \phi_{4}\right)^{\mathrm{T}}$ can be regarded as a vector composed of weights that are assigned to one or another component of the state vector $\beta$ when determining the short-term rate $y(0, \beta(t))=$ $=\beta^{\mathrm{T}}(t) \phi=\beta_{1}(t) \phi_{1}+\beta_{2}(t) \phi_{2}+\beta_{3}(t) \phi_{3}+\beta_{4}(t) \phi_{4}=r(t)$. In our case $\beta_{1}(t)+\beta_{4}(t)=r(t)$ according to (24), i.e. $\phi_{1}=1$, $\phi_{2}=0, \phi_{3}=0, \phi_{4}=1$. Substituting such bond price in equation (5), one can obtain a system of differential equations for the functions of the term structure $A(\tau)$ and $B(\tau)$ in the form

$$
\begin{gathered}
A^{\prime}(\tau)=(\sigma \lambda-K \theta)^{\mathrm{T}} B(\tau)+B(\tau)^{\mathrm{T}} \sigma \sigma^{\mathrm{T}} B(\tau) / 2, A(0)=0, \\
B^{\prime}(\tau)=\phi+K^{\mathrm{T}} B(\tau), B(0)=0 .
\end{gathered}
$$

Here $\lambda$ is a constant vector of market prices of risk. Solution of equation (33) is the following

$$
B_{1}(\tau)=\phi_{1} \frac{1-e^{-\gamma \tau}}{\gamma}, B_{2}(\tau)=\phi_{1} \frac{1-e^{-\gamma \tau}-\gamma \tau e^{-\gamma \tau}}{\gamma}+\phi_{2} \frac{1-e^{-\gamma \tau}}{\gamma}
$$




$$
B_{3}(\tau)=\phi_{3} \frac{1-e^{-\delta \tau}}{\delta}+\phi_{4} \frac{1-e^{-\delta \tau}-\delta \tau e^{-\delta \tau}}{\delta}, B_{4}(\tau)=\phi_{4} \frac{1-e^{-\delta \tau}}{\delta} .
$$

If we take into account that $\phi_{1}=1, \phi_{2}=0, \phi_{3}=0, \phi_{4}=1$ then the obtained functions of the term structure have no differences from the functions (23). The function $A(\tau)$ is equivalent to function $\tau \beta_{0}(\tau)$ as it follows from equations (19) and (32) because from definition $\sigma \lambda+K \theta=\lambda_{0}-\xi$, $\left(\sigma \lambda=\lambda_{0}, \xi=-K \theta\right)$.

So far we have considered the yield curve. As for the forward curve, it is linked in a one-to-one manner with the yield curve by the relations

$$
y(\tau, \beta)=\frac{1}{\tau} \int_{0}^{\tau} f(s, \beta) d s, \quad f(\tau, \beta)=y(\tau, \beta)+\tau \frac{\partial y(\tau, \beta)}{\partial \tau}
$$

or

$$
f(\tau, \beta)=\left(\tau \beta_{0}(\tau)\right)^{\prime}+\beta_{1}(t) \exp (-\gamma \tau)+\beta_{2}(t) \gamma \tau \exp (-\gamma \tau)+\beta_{3}(t) \delta \tau \exp (-\delta \tau)+\beta_{4}(t) \exp (-\delta \tau),
$$

which leads to the fact that function (34) also differs from (4) only by one additional term.

Since the result is an affine model of the time structure with a constant volatility matrix, the state variables are a four-dimensional stochastic process with a Gaussian distribution. We note that a detailed analysis of the properties of the Nelson-Siegel-Svensson model and its connection with affine Gaussian models is presented in [8] and [9].

Before proceeding to the conclusions, we cite an extensive quotation from [4. P. 89] which relates to the subject under consideration:

"The Filipović [10] critique of NS (Nelson-Siegel), namely that it would not be arbitrage-free if dynamized, also applies to the four-factor DNSS (Dynamic Nelson-Siegel-Svensson) model just introduced. One would hope that, in parallel to AFNS (ArbitrageFree Nelson-Siegel) and DNS (Dynamic Nelson-Siegel), one could derive an arbitrage-free approximation to DNSS. However, as the mechanics of Proposition AFNS of Chapter 3 make clear, an arbitrage-free Gaussian version of DNSS cannot exist, because it would require that each curvature factor be paired with a slope factor with the same rate of mean reversion, which is impossible with the single slope factor present in DNSS. (It is technically possible, however, to create an arbitrage-free version of DNSS if one leaves the Gaussian class, as in the model of Sharef and Filipovic [11], which has Cox et al. [12] square-root volatility.) Hence in the next subsection we add a second slope factor to match Svensson's second curvature factor, and we ask whether risk-neutral restrictions can be found that make the fivefactor model arbitrage-free."

The results described above do not fully coincide with the statements contained in this quote.

\section{Conclusion}

The following conclusions can be drawn from the text of the paper:

- The yield curve of the ECB (1) does not satisfy the no arbitrage conditions.

- To construct a no arbitrage yield curve, we need to add one more term in the same way as in the formula (22).

- As the state variables, it is necessary to choose factors $\left\{\beta_{1}, \beta_{2}, \beta_{3}, \beta_{4}\right\}$, which form a four-dimensional diffusion Gaussian process (30).

- The factor $\beta_{0}(\tau)$ is determined from the no arbitrage condition (28).

- Application of no arbitrage conditions to the Duffie-Kan model (with a square-root volatility), into which the Vasiček and Cox-Ingersoll-Ross models are being embedded, gives preference to the four-factor Vasiček model.

\section{REFERENCES}

1. Bank for International Settlements. (2005) Zero-Coupon Yield Curves: Technical Documentation. BIS papers. 25. pp. 1-55.

2. European Central Bank (2017) Euro area yield curves. [Online] Available from: https://www.ecb.europa.eu/stats/money/yc/html/index.en.html. 
3. European Central Bank. (n.d.) General description of ECB yield curve methodology. https://www.ecb.europa.eu/stats/financial_markets and interest_rates/euro_area yield_curves $/ \mathrm{html} / \mathrm{technical}$ notes.pdf?update $=20160513$

4. Diebold, F.X. \& Rudebusch, G.D. (2013) Yield curve modeling and forecasting: the dynamic Nelson-Siegel approach. Princeton, New Jersey: Princeton University Press.

5. Diebold, F.X. \& Li, C. (2006) Forecasting the Term Structure of Government Bond Yields. Journal of Econometrics. 130. pp. 337364. DOI: 10.1016/j.jeconom.2005.03.005

6. Vasiček, O.A. (1977) An Equilibrium characterization of the term structure. Journal of Financial Economics. 5. pp. 177-188. DOI: 10.1016/0304-405X(77)90016-2

7. Duffie, D. \& Kan, R. (1996) A yield-factor model of interest rates. Mathematical Finance. 6. pp. 379-406. DOI: 10.1111/j.14679965.1996.tb00123.x

8. Medvedev, G.A. (2015) On the Nelson-Siegel-Svensson no-arbitrage yield curve models. Vestnik Tomskogo gosudarstvennogo universiteta. Upravlenie, vychislitel'naya tekhnika i informatika - Tomsk State University Journal of Control and Computer Science. 3(32). pp. 44-55. (In Russian). DOI: 10.17223/19988605/32/5

9. Medvedev, G.A. (2015) The Nelson-Siegel-Svensson yields. Probability properties and estimation. Vestnik Tomskogo gosudarstvennogo universiteta. Upravlenie, vychislitel'naya tekhnika i informatika - Tomsk State University Journal of Control and Computer Science. 4(33). pp. 38-45. (In Russian). DOI: 10.17223/19988605/33/5

10. Filipovic, D. (1999) A Note on the Nelson-Siegel Family. Mathematical Finance. 9. pp. 349-359. DOI: 10.1111/1467-9965.00073

11. Sharef, E. \& Filipovic, D. (2004) Conditions for Consistent Exponential-Polynomial Forward Rate Processes with Multiple Nontrivial Factors. International Journal of Theoretical and Applied Finance. 7. pp. 685-700. DOI: 10.1142/S0219024904002608

12. Cox, J.C., Ingersoll, J.E. \& Ross, S.A. (1985) A Theory of the Term Structure of Interest Rates. Econometrica. 53. pp. $385-407$.

Medvedev Gennady Alexeevich, Dr. Sc., Professor. E-mail: MedvedevGA@bsu.by

Belarusian State University, Belarus.

Поступила в редакцию 23 июня 2017 г.

Медведев Геннадий Алексеевич (Белорусский государственный университет, Беларусь).

О кривых доходности Европейского центрального банка.

Ключевые слова: кривые доходности; форвардные кривые; модель Нельсона-Зигеля-Свенссона; условия отсутствия арбитража.

DOI: $10.17223 / 19988605 / 41 / 5$

Показано, что кривые доходности Европейского центрального банка не удовлетворяют условиям отсутствия арбитража. Чтобы сконструировать безарбитражную кривую доходности, необходимо добавить еще одно слагаемое к существующему выражению кривой доходности. Что касается переменных состояния, необходимо использовать четырехмерный гауссовский диффузионный процесс. Пятый фактор определяется из условий отсутствия арбитража. Предлагаемая версия модификации кривой доходности отличается от похожих модификаций, ранее предложенных в литературе, для гарантирования отсутствия арбитражных возможностей. Применение условий отсутствия арбитража к модели Даффи-Кана (с волатильностью с квадратным корнем), в которую вложены модели Васичека и Кокса-Ингерсолла-Росса, отдает предпочтение четырехфакторной модели Васичека. 